\title{
L-Arginine Protective Effect on Systemic Blood Pressure and Placental Expression of Endoglin, Transforming Growth Factor- $\beta 1$ in the Preeclampsia Mice Model
}

\author{
Manggala Pasca Wardhana ${ }^{*(\mathbb{D}}$, Budi Wicaksono $^{1}$, Aditiawarman $^{1}$, Widjiati $^{2}$, Muhammad Ardian' $^{1}$, \\ Meilia Dwi Cahyani ${ }^{3}$ Rizqy Rahmatyah ${ }^{3}$
}

\begin{abstract}
Objectives: Research on understanding the pathogenesis of preeclampsia and its alternative management is still not optimal, resulting in high morbidity and mortality, especially in developing countries. Therefore, this study aimed to understand the L-arginine effect on placental endoglin, transforming growth factor-ß1 (TGF-ß1) expression and blood pressure in preeclampsia.

Materials and Methods: Using a post-test control group design, this randomized experimental study was carried out on an anti-Qa2 preeclampsia mice model, in which the treatment group $(n=9)$ was given L-arginine orally. Blood pressure was measured on day 15 and mice were terminated at day 16 to evaluate endoglin and TGF-B1 placenta expression through immunohistochemistry.

Results: Based on the results, lower systolic and diastolic blood pressure $(P<0.05)$ and significantly lower endoglin and higher TGF-ß1 placental expression were found in the L-arginine treatment group compared with the control (the preeclampsia mice model) group $(P<0.05)$.

Conclusions: In general, L-arginine can ameliorate systemic blood pressure and locally in the placenta by providing lower expression of endoglin while higher TGF-ß1 in the preeclampsia mice model.

Keywords: L-arginine, Blood Pressure, Endoglin, Transforming Growth Factor-ß1, Preeclampsia
\end{abstract}

\section{Introduction}

Preeclampsia is a significant contributor to maternal morbidity and mortality, especially in developing countries. In general, $10 \%$ of pregnancies have complications of preeclampsia (1). Wardhana's study from one of the tertiary services in eastern Indonesia demonstrated an up to twice prevalence that of $21 \%$ (1106 cases) for two years. The high maternal mortality rate in Indonesia (359 in 2012) shows that this condition is still a big problem in developing countries (2).

One of the characteristics of preeclampsia is the occurrence of global vasoconstriction, which shows the possibility of a decrease in NO endothelial production. Studies have indicated that a disorder of transforming growth factor beta 1 (TGF- $\beta 1$ ) caused by its binding to sEng can interfere with the activation of nitric oxide synthase (NOS) to produce nitric oxide (NO), along with the vessel vasodilation and angiogenesis leading to vasoconstriction and endothelial dysfunction as the characteristics of preeclampsia (3).

L-arginine, as a NO precursor, is a semi-essential amino acid in which production is not enough when the NO requirement increases as in some conditions of pregnancy with specific pathologies. The concentration of L-arginine is known to decrease significantly in preeclampsia compared to normal pregnant women who show the potential for NO production disturbances (4). $\mathrm{L}$-arginine has the role of producing $\mathrm{NO}$ as a vasodilator to increase placental blood flow so that it can improve the shallow condition of placental implantation, angiogenesis, and vasoconstriction. However, this hypothesis still requires further research to ensure a better pathogenesis pathway. It is fascinating to know the role of L-arginine in the systemic clinical parameters of preeclampsia such as blood pressure and its local pathogenic pathways in the placenta through the examination of Eng and TGF- $\beta 1$.

\section{Materials and Methods}

Research Subject

True experimental, randomized post-test only, control group research was done in female healthy adult Balb/c mice (Mus musculus) obtained from The Integrated Research and Testing Laboratory, Gajah Mada University, Indonesia. Eighteen healthy, pregnant mice of 3 months old with 20-30 g were included in this study and randomized into preeclampsia model/control and preeclampsia model with L-arginine intervention groups. All groups were given the same foods, beverages, and cage conditions. We 


\section{Key Message}

- Evaluation of placental biomolecular conditions in preeclamptic conditions with the L-arginine intervention has not been found yet.

- Significant differences were found in the expression of placental endoglin and TGF-ß1 from preeclamptic mice which can be a sign of placental improvements with the L-arginine intervention.

did blood pressure measurement on day 15 and then did the termination on day 16 .

\section{Mating Procedure}

Three-month female mice $(20-30 \mathrm{~g})$ did mating procedures by the Practice Committee of the American Society for the Reproductive Medicine procedure (5). Estrus synchronization with $5 \mathrm{IU}$ pregnant mare's serum gonadotropin (PMSG PG 600, MSD Animal Health, Intervet Inc.) was injected and continued with 5 IU human chorionic gonadotropin (hCG Chorulon, MSD Animal Health, Intervet Inc.) injection 48 hours later. Monomating was done for all mice (male mice, seven months, $40 \mathrm{~g}$ ), and 17 hours later, the pregnancy was diagnosed by the presence of copulatory plug as day 0 of gestation.

\section{Preeclampsia Model Preparation}

The Sulistyowati method was used to make a preeclampsia mice model by injecting ten ng anti-Qa2 intraperitoneally (the analog of anti-HLA-G) on both groups from the 1st until the fourth day (6). Anti-Qa2 will diminish HLA-G expression to induce the preeclamptic mice model. With a slope of $45^{\circ}$ and $0.5 \mathrm{~cm}$ deep, the $27 \mathrm{G}$ needle was intraperitoneally injected in the upper right abdomen (6).

\section{L-arginine Intervention}

The L-arginine $200 \mathrm{mg} / \mathrm{kg} / \mathrm{d} \mathrm{BW}$ was orally given by the stomach tube from day 6 until 14 to the intervention group. Then, saline $(0.1 \mathrm{~mL} / 10 \mathrm{~g})$ was orally given as a placebo to the control mice (7).

\section{Blood Pressure Examination}

On day 15, all mice underwent blood pressure measurement (systolic and diastolic) using tail-cuff by specialized volume pressure recording (MBRP-MMC, IITC Life Science Inc, CA, USA) according to (8). All the exam preparation was done to avoid stress during blood pressure examinations.

Endoglin and Transforming Growth Factor- $\beta 1$ Immunohistochemistry Examination

The placental tissue was fixed with $7 \%$ buffered formalin while preparing the paraffin section. For TGF- $\beta 1$ expression examinations, 1:100 dilution of anti-TGF- $\beta 1$ (MAB1673; R\&D Systems) was given on the slide and incubated at room temperature for 1 hour. The same process was also done to the endoglin examination, and 1:100 dilution of anti CD 105/endoglin (MAB1320, R\&D Systems) was given for immunohistochemistry examinations.

TGF- $\beta 1$ and Eng expression were observed in 10 random high-power fields with 400x magnification. A modified semi-quantitative immunoreactive score (IRS) was used by Remmele and Stegner based on the work of Nowak with a Nikon H600L light microscope. The positive expression is shown by the red-brown chromogen. This method relied on both the percentage of positive cells and the intensity of the color reaction. The final score (ranging from 0 to 12 points) was represented as no reaction: 0 pts $(-)$, poor reaction: $1-2$ pts $(+)$, moderate reaction: $3-5$ pts $(++)$, and intense reaction: 6-12pts $(+++)$.

\section{Statistical Analysis}

SPSS software (version 24.0) for Windows (IBM Corp., Armonk. N.Y., USA) was used for statistical analysis. Continuous variables were reported using the mean \pm standard deviation. In addition, an independent t-test and Mann-Whitney test as an alternative were applied to compare the difference in the continuous variables between the group, and a $P<0.05$ was considered statistically significant.

\section{Results}

The Effect of L-arginine on Blood Pressure

The anti-Qa2 was given to establish a preeclampsia mice model and L-arginine was given to the intervention group. Blood pressure was checked on the 15th day, and a significantly lower blood pressure was found in systolic (163.55 $\pm 10.46 \mathrm{mmHg}$ vs. $143.55 \pm 14.88 \mathrm{mmHg} P=0.015)$ and diastolic (116.44 $\pm 23.51 \mathrm{mmHg}$ vs. $144.33 \pm 12.06$ $\mathrm{mmHg}, P=0.021$ ) blood pressures of the treatment group compared to the control group (Figure 1).

\section{The Effect of L-arginine on Placental Endoglin}

Placental ischemia disturbance has already known as one of the pathogenesis pathways in preeclampsia. In this study, endoglin was evaluated to determine the effect of $\mathrm{L}$-arginine on the improvement of this pathogenesis. The expression of placental endoglin in Figure 2 (A1 and A2) is shown in brownish-red. The modified IRS assessed the degree of endoglin expression for ten fields of view, which resulted in significantly lower endoglin expression in the treatment group compared with the control group (Figure 3).

The Effect of L-arginine on Transforming Growth Factor- $\beta 1$

Considering the modulation of TGF- $\beta 1$ by its binding, endoglin can disturb angiogenesis and the vasodilatation process that has an essential role in the pathogenesis of preeclampsia. Accordingly, the current study evaluated the 

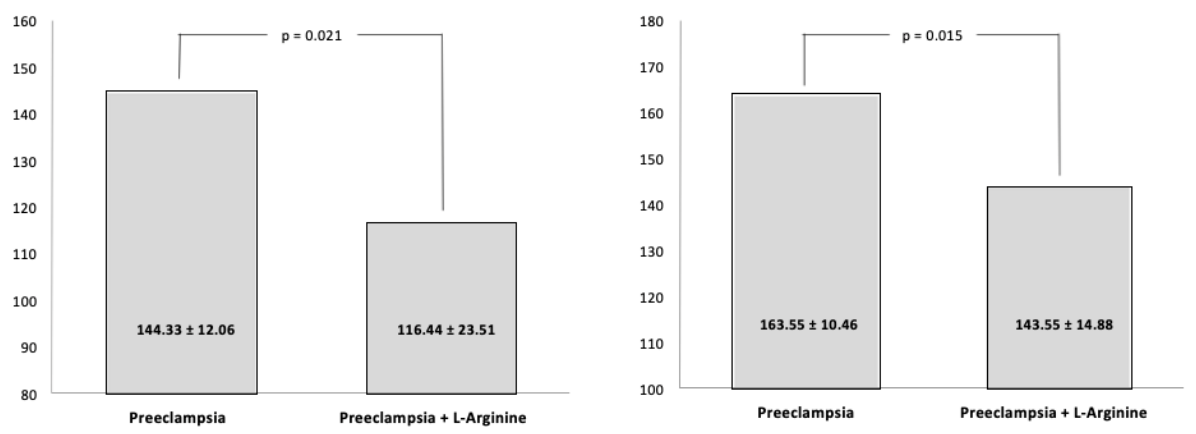

Figure 1. Systolic and Diastolic (mean \pm SD) Blood Pressure on Day 15 of Gestation. Note. SD: Standard deviation. The intervention group had lower systolic and diastolic blood pressure compared to the control group $(P<0.05)$.

placental expression of TGF- $\beta 1$ to identify the protective effect of L-arginine locally in the placenta. The expression of placental endoglin can be observed in Figure 2 (B1 and B2) in brownish-red. A modified IRS assessed the degree of TGF- $\beta 1$ expression for ten fields of view, leading to significantly higher TGF- $\beta 1$ expression in the treatment group in comparison with the control group (Figure 3).

\section{Discussion}

In the pathology of the preeclamptic placenta, an examination is needed when this disorder arises after the second trimester and spiral artery remodeling represents a termination. Research on the human placenta can only be done in cases of miscarriage in the early trimester before the onset or at the termination of preeclampsia where clinical symptoms have occurred far after a pathological disorder in preeclampsia and can have a bias from the pathologies of the labor itself and the difference of gestational age (10). Therefore, in this study, the animal model was selected to find the problems that cannot be explained in human research due to ethical issues.

Accordingly, the anti-Qa2 mice preeclampsia model was chosen to establish a model of preeclampsia since

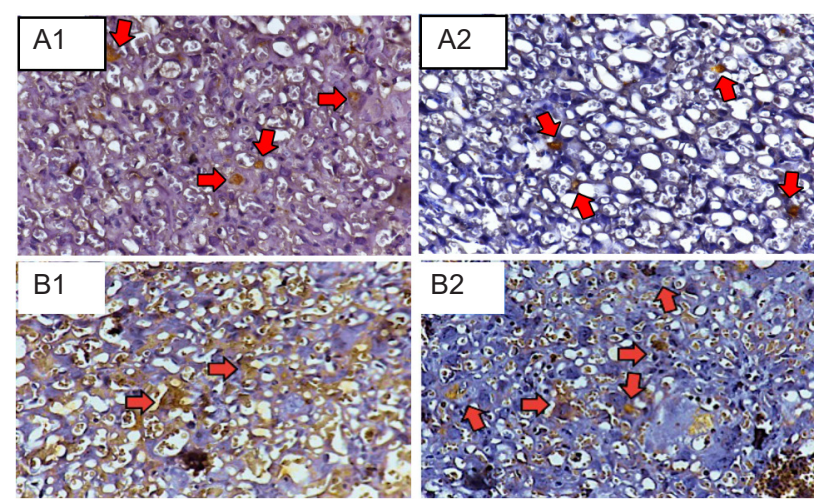

Figure 2. Expression of Placental Endoglin dan TGF-ß1. Note. TGF-ß1: Transforming growth factor-B1; SD: Standard deviation. (A) Expression of placental endoglin: (1) Control and (2) treatment groups. (B) Expression of placental TGF- $\beta 1$ : (1) Control and (2) treatment groups. Expression in trophoblast cells is shown by red arrows (x4000 magnification, Nikon $\mathrm{H} 600 \mathrm{~L}$ microscope, 300 megapixel DS camera). the beginning of its pathogenesis so that to study various pathological conditions that locally occur in the placenta. Anti-QA2 was provided as an anti-HLA-G equivalent to diminish its expression in the trophoblast (6). Various pathogenesis pathways of preeclampsia can occur with the loss of HLA-G expression, ranging from the disruption of trophoblast invasion, and the remodeling of spiral arteries which continue with the occurrence of placental ischemia until giving systemic symptoms as increased blood pressure and proteinuria. Several studies of other preeclamptic mouse models used different methods such as providing uteroplacental ischemia through the tie-up of the abdominal aorta, reduction of continuous uteroplacental circulation, anti-NOS administration, and the administration of antiangiogenesis and various other methods which generally do not directly provide pathological conditions in the trophoblast as in our study.

The results of this study showed significantly lower blood pressure on both systolic and diastolic pressures in the intervention group as compared with the control group, which is in line with the results of the research by Alexander, which examined the preeclampsia mice with a different model using a reduced uterine perfusion pressure model. In this model of mice, an increase was observed in blood pressure and this increase could be significantly $(P<0.01)$ reduced by the administration of $\mathrm{L}$-arginine (9). Several L-arginine intervention studies on humans with preeclamptic conditions vary greatly regarding their protocol. Some of these studies provide acutely high doses in a short time while some of them provide long-term administrations as well as the method presented in this study. The study by Rytlewski et al (10) provided L-arginine for a long time orally for three weeks, which is in conformity with the results of the present study. Systolic $(134.2 \pm 2.9$ vs. $143.1 \pm 2.8)$ and diastolic (81.6 \pm 1.7 vs. $86.5 \pm 0.9)$ blood pressures were significantly lower $(P<0.01)$. However, Neri et al found no significant difference regarding blood pressure in pregnancy cases with chronic hypertension was observed and without L-arginine although the positive results of using L-arginine in the above-mentioned study included a decrease in the use of antihypertensive drugs whereas 

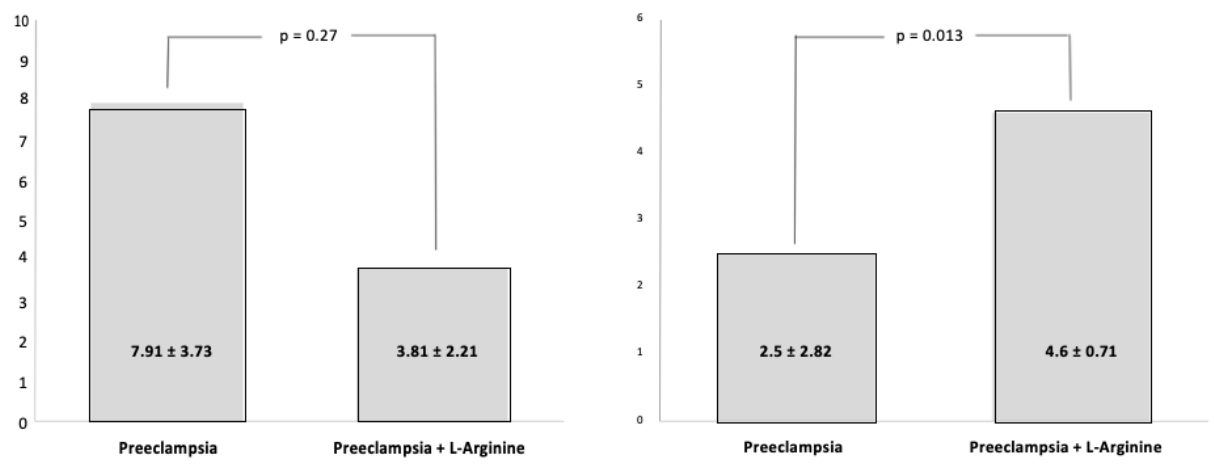

Figure 3. Immunoreactive Score Results of Placental Endoglin and TGF-ß1 Expression (mean \pm SD)

Note. TGF-ß1: Transforming growth factor-ß1; SD: Standard deviation. The treatment group had a significant decrease in endoglin while an increase in TGF-ß1 placental expression $(P<0.05)$.

more anti-hypertension was needed in the placebo group so that in human studies, observing direct differences in blood pressure resulting from the use of L-arginine was generally difficult (11).

Although not carried out in this study, several other studies mentioned the antihypertensive effect of L-arginine associated with increased NO release, which correlates with a decrease in blood pressure while an increase in L-Citrulline. The results of the study by Rytlewski et al also showed that the results of significantly lower blood pressure measurement on L-arginine administration could be due to the increase in NO demonstrated in the study with significantly higher nitrate/nitrite production $(P<0.05)$ and $\mathrm{L}$-ornithine $(P<0.05)$ in patients receiving $\mathrm{L}$-arginine (10). The administration of L-arginine seems to be quite promising for reducing blood pressure, as indicated by several studies that showed L-arginine has antihypertensive effects; even though some other studies demonstrated controversial results (12). Based on the results of this study, the condition of a significant decrease in the blood pressure that occurs on systolic and diastolic blood pressure with the administration of L-arginine can be one of the modalities for the management of preeclampsia. Human research needs to be done with proper quality methods and designs to prevent various biases, which is difficult to control in social research.

On immunohistochemical examination under a microscope against placental mice, the endoglin and TGF- $\beta 1$ expression observed in trophoblast cells spread over the placental tissue. The results of this study revealed a significantly lower endoglin and higher TGF- $\beta 1$ placental expression in the group of preeclamptic mice who received L-arginine compared to the control group. Although there are various L-arginine studies on systemic blood pressure conditions, few have locally focused on their effects on the placenta. To the best of our knowledge, this is the first study to determine the effect of L-arginine on several placental pathologies associated with the pathogenesis of preeclampsia.

Endoglin is a cell surface coreceptor of TGF- $\beta 1$ and
TGF- $\beta 3$ produced by trophoblast and endothelial cells for modulating the activity of TGF- $\beta 1$ and TGF- $\beta 3$ which are able to provide antiangiogenic environmental conditions (13). Venkatesha et al found a 4-fold increase in the endoglin mRNA of the preeclamptic placenta confirmed by the increased expression of immunostaining from the placental section (14). Another study by Jeyabalan et al (15) also showed a significant increase in placental endoglin in placenta preeclampsia compared to normal $(14.4 \pm 2.7$ vs $5.7 \pm 0.9, P=0.01)$.

The increasing of endoglin intensifies the TGF- $\beta 1$ modulation. Several factors such as sEng and sFlt-1 increase by an increase in the production of anti-angiogenic molecules in preeclampsia caused by placental hypoxia. sEng interacts with TGF- $\beta 1$ which causes a reduction in the action of TGF- $\beta 1$ on the surface receptor. This will inhibit angiogenesis and apoptosis leading to endothelial dysfunction. Similarly, Venkatesha et al demonstrated a decrease of TGF- $\beta 1$ in preeclampsia resulting from an increase in sEng showed by a significant decrease in vascular diameter and vasoconstriction. In addition, sEng binds to TGF- $\beta 1$ and competes with the TGF- $\beta$ receptor in the endothelium. Finally, the decrease of TGF- $\beta 1$ caused by its binding to sEng has an implication for the activation of eNOS by interfering with the dephosphorylation of Thr495 which can globally reduce NO production and disturb blood vessel angiogenesis and vasodilation (14).

The effect of giving L-arginine to endoglin and TGF- $\beta 1$ in placental preeclampsia itself has not been studied yet. The mentioned L-arginine can improve the clinical condition of preeclampsia through blood pressure, along with improving placental endoglin and TGF- $\beta 1$ conditions in this study which might have an influence on the NO vasodilation pathway. A systematic review by Dorniak-Wall (16) represents the ability of L-arginine for the prevention of preeclampsia. L-arginine is associated with a reduction in preeclampsia compared with placebo risk ratio of 0.34 (95\% CI: 0.21-0.55). Further, L-arginine can be a promising therapy although information about its role in maternal and infant health outcomes is still limited. 
The disturbance of spiral artery remodeling, as well as global vascular endothelial dysfunction is a condition that occurs in preeclampsia. Accordingly, the role of $\mathrm{NO}$ as a protective blood vessel factor synthesized from the amino acid L-arginine cannot be ruled out naturally. NO has the ability of vasodilation associated with the inhibition of thromboxane production, platelet aggregation, and the stimulation of prostacyclin production. NO donor is $\mathrm{L}$-arginine, thus the condition of reduced L-arginine will be associated with the occurrence of hypertension in pregnancy and preeclampsia in some studies (16). Furthermore, NO has an important role in the obstetric field, and the NO inducing relaxation of the smooth muscle, the main organ for NO production is endothelium with L-arginine as its substrate. Thus, the availability of these amino acids is highly important as an adaptive regulator that counteracts vasoconstriction conditions in preeclampsia. L-arginine is a semi-essential amino acid, where the need for high endogenous synthesis is insufficient for meeting its needs. The disorders of synthesis from NO are also shown in preeclampsia. Evidence showed that the availability of L-arginine as a supplement can help vasodilation through the increased production of NO. Arginine can be insufficient in preeclampsia. Asymmetric dimethylarginine concentration as a competitive inhibitor of NOS is also known to be increased in preeclamptic women. Moreover, some other disorders occur through an increased mechanism of Endoglin which can inactivate NOS through the modulation of TGF- $\beta 1$ (17).

The finding of this study showed the significant role of L-arginine in the endoglin and TGF- $\beta 1$ placenta production which provides evidence that the role of L-arginine not only systemically improves blood pressure but also is thought to occur at the placental level which also helps in improving angiogenesis and placental vascularization even in the conditions of shallow trophoblast invasion through this mice model of preeclampsia. In another study on mice, Al-Bayati et al reported that L-arginine can significantly increase placental weight $(P<0.05)$ which can be due to the effect of $\mathrm{NO}$ on the placenta resulting in dilated placental villous vascularization. NO can be found in the Vilous region affecting vascular tone by inhibiting the vasoconstrictors such as endothelin and thromboxane. In addition to placental weight, the size, volume, and surface of the placenta increased significantly $(P<0.05)$ by the administration of L-arginine which could be due to the effect of increasing NO production on trophoblast cells thereby increasing vascular vasodilation (7).

In line with this study and that of Al-Bayati et al, along with causing a downstream improvement in preeclampsia as indicated by lower blood pressure in this study, it is suspected that this treatment can improve the vascularization condition in the placenta by the administration of L-arginine. The study by $\mathrm{Wu}$ on sows given the arginine diet showed an increase in mean birth weight, VEGFA expression, and PlGF1 mRNA expression. This significant increase demonstrated the important role of the arginine diet in placental vascular function and the supply of nutrients to the fetus (18). The increased uteroplacental circulation due to the effect of $\mathrm{NO}$ on angiogenesis and vasodilation affected by the administration of exogenous L-arginine will certainly improve the condition of placental ischemia so that it may reduce antiangiogenic production in preeclampsia such as endoglin. As a result of this decrease in production, the effect of the modulation of sEng on TGF- $\beta 1$ will decrease, and finally, it can increase its expression in the placenta.

Further research is needed to look at pathogenesis pathways that can cause a decrease in Endoglin while an increase in TGF- $\beta 1$, as was observed in this study. In this study, preeclamptic model animals were used which can be different if done on humans, thus confirmation studies conducted on humans with good methods will reveal a thorough understanding of the effect of L-arginine on improving preeclamptic placenta, one of which in this study was demonstrated by the decrease in endoglin while an increase in TGF- $\beta 1$ expression. Several weaknesses were found in this study. One of them was blood pressure measurement carried out outside the city, where these conditions can interfere with different stress levels in mice and can certainly affect measurement results. The obtained decrease in endoglin and an increase in TGF- $\beta 1$ expression in this study need to be confirmed to identify exactly whether it is because of the direct effect of the product of L-arginine, $\mathrm{NO}$ in the placenta. It would be better if both were examined in the placenta of mice in this study. The pre and post design will also be better to identify the effect of L-arginine intervention in this study. However, it was difficult since this research need placental samples which are impossible to obtain before the termination.

\section{Conclusions}

L-arginine has a protective role in preeclampsia. Our results revealed a lower level of both systemic systolic and diastolic blood pressures in the intervention group compared with the control group. L-arginine may also have a locally good influence on the placenta indicated by lower endoglin and higher TGF- $\beta 1$ expression which might ameliorate both NO production and the improvement of the preeclamptic placenta. Although the $\mathrm{L}$-arginine pathway as a preeclamptic treatment still needs to be investigated, this intervention might be promising alternative management of preeclampsia because it can improve the overall condition of preeclampsia.

\footnotetext{
Authors' Contribution

MPW, BW, A, W and MA contributed to research conceptualization. MDC and RR contributed to application for ethics review and approval. MPW and MA contributed to data collection. MPW, MDC and RR drafted the manuscript and submitted for publication. All authors contributed to analysis and discussions of situations.
} 


\section{Conflict of Interests}

Authors declare that they have no conflict of interests.

\section{Ethical Issues}

The study was approved by the Ethics Committee of the Veterinary Medicine Faculty, Universitas Airlangga.

\section{Financial Support}

Our research was supported by a research grant from Airlangga University (1408/UN3/2019).

\section{References}

1. RobertsJM,AugustPA, Bakris G, etal.Hypertension in pregnancy: Executive summary. Obstet Gynecol. 2013;122:1122-31. doi: 10.1097/01.AOG.0000437382.03963.88

2. Wardhana MP, Dachlan EG, Dekker G. Pulmonary edema in preeclampsia: an Indonesian case-control study. J Matern Fetal Neonatal Med. 2018;31:689-95. doi: 10.1080/14767058.2017.1295442

3. López-Jaramillo P, Arenas WD, García RG, Rincon MY, López M. The role of the L-arginine-nitric oxide pathway in preeclampsia. Therapeutic advances in cardiovascular disease 2008; 2: 261-75. doi: 10.1177/1753944708092277

4. McCord N, Ayuk P, McMahon M, Boyd RC, Sargent I, Redman C. System y+ arginine transport and NO production in peripheral blood mononuclear cells in pregnancy and preeclampsia. Hypertension. 2006;47:109-15. doi: 10.1161/01.HYP.0000197952.22711.c4

5. Practice Committee of American Society for Reproductive Medicine, Birmingham, Alabama. Gonadotropin preparations: past, present, and future perspectives. Fertil Steril. 2008;90(5 Suppl):S13-20. doi: 10.1016/j.fertnstert.2008.08.031.

6. Sulistyowati S, Bachnas MA, Anggraini ND, et al. Recombinant vascular endothelial growth factor 121 injection for the prevention of fetal growth restriction in a preeclampsia mouse model. J Perinat Med. 2017;45:245-51. doi: 10.1515/jpm2016-0149

7. Al-Bayati MA, Ahmad MA, Khamas W. The potential effect of L-arginine on mice placenta. Adv Pharmacoepidemiol Drug Saf. 2014;3:1-9. doi: 10.4172/2167-1052.1000150

8. Wang Y, Thatcher SE, Cassis LA. Measuring blood pressure using a noninvasive tail cuff method in mice. The ReninAngiotensin-Aldosterone System. Springer; 2017. p. 69-73. doi: 10.1007/978-1-4939-7030-8_6

9. Alexander BT, Llinas MT, Kruckeberg WC, Granger JP. L-arginine attenuates hypertension in pregnant rats with reduced uterine perfusion pressure. Hypertension 2004;43: 832-6. doi: 10.1161/01.HYP.0000119192.32360.a9.

10. Rytlewski K, Olszanecki R, Korbut R, Zdebski Z. Effects of prolonged oral supplementation with L-arginine on blood pressure and nitric oxide synthesis in preeclampsia. Eur J Clin Invest. 2005;35:32-7. doi:10.1111/j.1365-2362.2005.01445.x

11. Neri I, Monari F, Sgarbi L, Berardi A, Masellis G, Facchinetti F. L-arginine supplementation in women with chronic hypertension: impact on blood pressure and maternal and neonatal complications. J Matern Fetal Neonatal Med. 2010; 23:1456-60. doi: 10.3109/14767051003677962

12. Gui S, Jia J, Niu X, et al. Arginine supplementation for improving maternal and neonatal outcomes in hypertensive disorder of pregnancy: a systematic review. J Renin Angiotensin Aldosterone Syst. 2014;15:88-96. doi: $10.1177 / 1470320313475910$

13. Cheifetz S, Bellón T, Calés $C$, et al. Endoglin is a component of the transforming growth factor-beta receptor system in human endothelial cells. J Biol Chem. 1992;267:19027-30.

14. Venkatesha S, Toporsian M, Lam C, et al. Soluble endoglin contributes to the pathogenesis of preeclampsia. Nature Med. 2006;12:642-9. doi: 10.1038/nm1429

15. Jeyabalan A, McGonigal S, Gilmour C, Hubel C, Rajakumar A. Circulating and placental endoglin concentrations in pregnancies complicated by intrauterine growth restriction and preeclampsia. Placenta. 2008;29:555-63. doi: 10.1016/j. placenta.2008.03.006

16. Dorniak-Wall T, Grivell R, Dekker G, Hague W, Dodd J. The role of L-arginine in the prevention and treatment of preeclampsia: a systematic review of randomised trials. J Hum Hypertens. 2014;28:230-5. doi: 10.1038/jhh.2013.100

17. Hedge C. The use of L-arginine in the management of preeclampsia and intruterine growth restriction. J Obstet Gynaecol India. 2012;62:1-2. doi: 10.1007/s13224-0120146-8.

18. Wu $X$, Yin $Y$, Liu $Y$, et al. Effect of dietary arginine and $\mathrm{N}$-carbamoylglutamate supplementation on reproduction and gene expression of eNOS, VEGFA and PIGF1 in placenta in late pregnancy of sows. Anim Reprod Sci. 2012;132:187-92. doi: 10.1016/j.anireprosci.2012.05.002

C 2021 The Author(s); This is an open-access article distributed under the terms of the Creative Commons Attribution License (http:// creativecommons.org/licenses/by/4.0), which permits unrestricted use, distribution, and reproduction in any medium, provided the original work is properly cited. 